\title{
Lateral wind force and torque estimation for a driving assistance ${ }^{\star}$
}

\author{
Sébastien Glaser* Saïd Mammar** Djamil Dakhlallah* \\ * LIVIC / LCPC, Versailles France (e-mail: name@lcpc.fr) \\ ** IBISC-CNRS-FRE 2873, Evry France (e-mail: \\ said.mammar@iup.univ-evry.fr)
}

\begin{abstract}
Main topics of driving assistances with respect to degraded driving situations, are low friction or low visibility. Thus, wind related problems are often less considered. However on high bridges or near sea shores, wind can generate car accident. In fact, wind shall have a huge impact on vehicle dynamic. For light vehicle, it could surprise the driver, resulting in a lateral movement. Looking at heavy truck, wind can be an additional factor that initiate a rollover. In this paper, an observer based method for the estimation of wind impact on vehicle dynamic is proposed. It develops the problem of numerical observability of the wind impact and propose an architecture to measure it. Next, it uses this knowledge in order to build a safe speed profile.
\end{abstract}

\section{INTRODUCTION}

Wind influence on vehicle dynamic is various, depending on the vehicle type. For passenger vehicle, a strong variation on lateral wind may surprise the driver, resulting in a lateral movement on the lane. This movement could be large enough to go outside of the lane. In the case of a truck, the wind shall generate an additional force that will initiate a rollover.

However, the impact on the lateral dynamic mostly depend on the transition between a state with low, or no, wind the a windy situation. This appears on many roads, in mountain for instance, where you have high bridges, or near sea shores with covered area.

The cover could be a static object, for instance a wall, a house or a tree lane, or a dynamic object, a truck with a long lorry for instance.

Whatever the cover, the driver may be surprised and, given his work load at this moment, he will react after a given time $T_{r}$. During this period, the vehicle will move laterally to the lane and could cross the lanes. A system that warn him earlier on lateral wind could reduce this lateral movement.

The problem of wind estimation is often tackled from the point of view of air vehicle [Eld06, Kum05]. But, in the case of air vehicle, the wind has the most impact on the general dynamic. In the case of a car, or a truck, the wind is mainly seen as a disturbance that a control will reject [Cor04]. Our objective is to develop an estimation of the wind impact on the vehicle dynamic.

The sequel of this article is organized as follow. In the first section, we will briefly introduce the forces and torques generated by the wind. Section two is devoted to the vehicle model and impact of a lateral wind on the vehicle. Next section will develop the wind estimator.

\footnotetext{
‡ This work was supported by the French National Research Agency, through SARI and DIVA projects
}

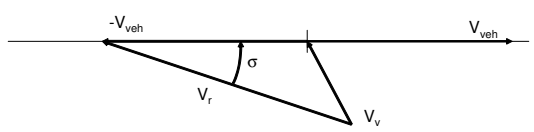

Fig. 1. Vehicle Relative wind speed

\section{WIND MODEL}

This section will present the model used for the impact of the wind on the vehicle dynamic. It will also present hypothesis done in order to simplify the model.

\subsection{Wind forces and torques}

Wind forces are applied all over the vehicle. The resulting force and torque mainly depend on the wind and vehicle shape. However, [Bak87], both forces and torques generated by the wind on the vehicle could be summarized in a set of force and torque applied at the center of gravity of the vehicle given the three axes : longitudinal, lateral and vertical. General expression of forces on the $i$ axes is:

$$
F_{i}=\frac{1}{2} C_{i} \rho S V_{r}^{2}
$$

For the torque, the expression is:

$$
M_{i}=\frac{1}{2} C_{i} \rho S E V_{r}^{2}
$$

In these equations:

- $C_{i}$ are adimensional coefficients describing the vehicle and the wind direction,

- $V_{r}$ is vehicle relative wind speed (see fig. 1),

- $S$ is the area of reference for the vehicle,

- $E$ is the reference length,

- $\rho$ is air density, $\rho=1.292$.

$E$ and $S$ depend on the vehicle, and Baker [Bak87] has developed an empiric model giving a relation between the $C_{i}$, the wind and the vehicle:

- $C_{x}=-a_{3}(1+2 \sin (3 \sigma))$, 


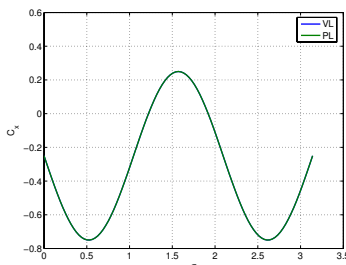

(a)

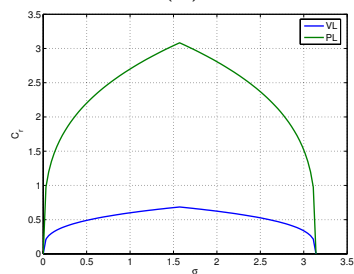

(c)

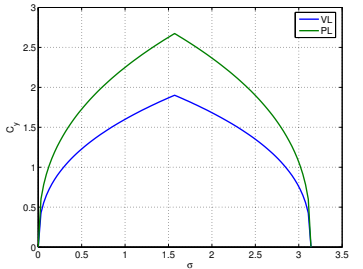

(b)

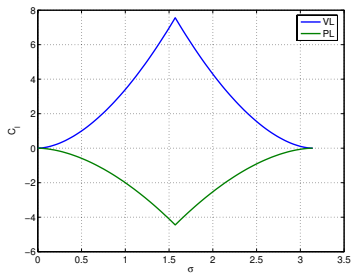

(d)
Fig. 2. $C_{i}$ for passenger car $(\mathrm{VL})$ and heavy truck $(\mathrm{PL})(\sigma$ is in $\mathrm{rad}$ )

- $C_{y}=a_{1} \sigma^{0.382}$,

- $C_{z}=-a_{2}(1+\sin (3 \sigma))$

- $C_{r}=a_{6} \sigma^{0.294}$,

- $C_{t}=a_{5} \sigma^{1.32}$

- $C_{l}=-a_{4} \sigma^{1.77}$.

Three last coefficients are the coefficients of the torques around respectively the longitudinal, lateral and vertical axes. $a_{i}$ coefficients depend on the vehicle.

\subsection{Impact on vehicle dynamic}

For both passenger car and heavy vehicle, the figures 2.(a) to (d) show the values of main $C_{i}$ according to the vehicle relative wind direction.

Even if the $C_{x}$ are similar for passenger vehicle and heavy truck, the force generated is different because of the reference length that varies. Moreover, values of $C_{x}$ (resp. $C_{r}$ ) are far below the values of $C_{y}$ (resp. $C_{l}$ ). In this study, we will simplify wind impact to the lateral forces and yaw rate torques.

\section{VEHICLE MODEL}

This section aim at analyzing the impact of wind on the vehicle and next, to present the model used in order to estimate lateral wind.

\subsection{Wind impact on dynamic}

As our goal is to show vehicle loss of control, model used in this section must be able to have a high dynamic and could simulate non linear behavior. The chosen model is a four wheeled vehicle, without suspension and with non linear model of tire road contact using a Dugoff model [Dug77]. This tire road model presents the advantage to have few parameters to described the force, and still keeping the main effect of vertical load, road friction and tire stiffness. The vehicle model is then embedded with a driver model that regulate both longitudinal [Gla07] and lateral dynamic [Net03]. The lateral control have been tuned up in order to correct a lateral displacement of $1 \mathrm{~m}$ in 1s. Wind speed on both longitudinal and lateral axes
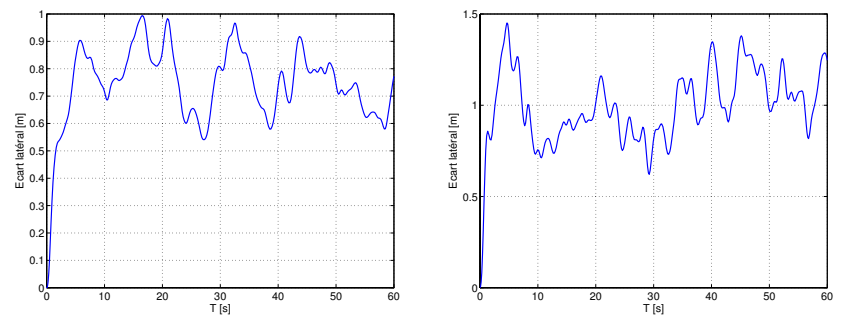

Fig. 3. Lateral displacement for a vehicle driving at $15 \mathrm{~m} . \mathrm{s}^{-1}$ (left) and $30 \mathrm{~m} . \mathrm{s}^{-1}$ (right)
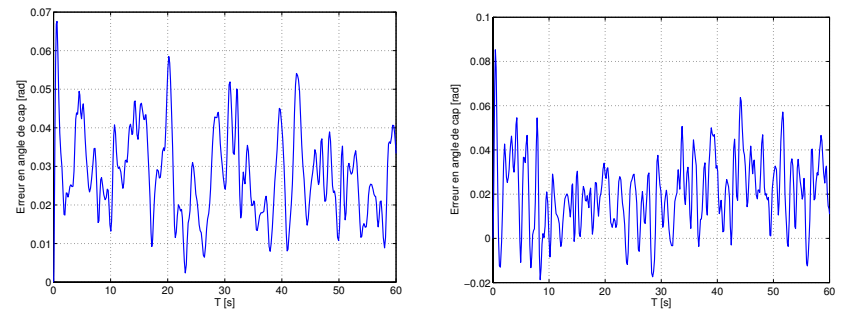

Fig. 4. Yaw angle error for a vehicle driving at $15 m . s^{-1}$ (left) and 30 m.s $s^{-1}$ (right)
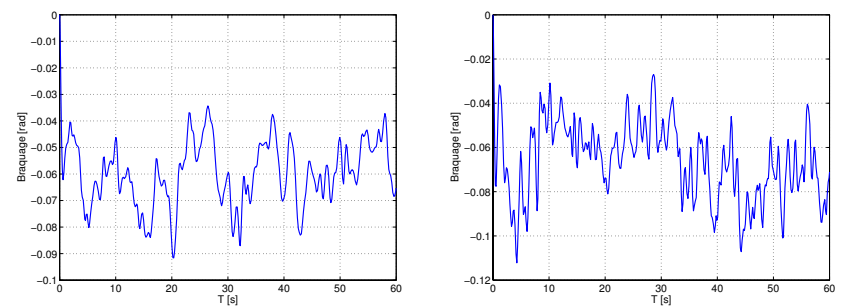

Fig. 5. Steering angle for a vehicle driving at $15 \mathrm{~m} . \mathrm{s}^{-1}$ (left) and $30 m . s^{-1}$ (right)
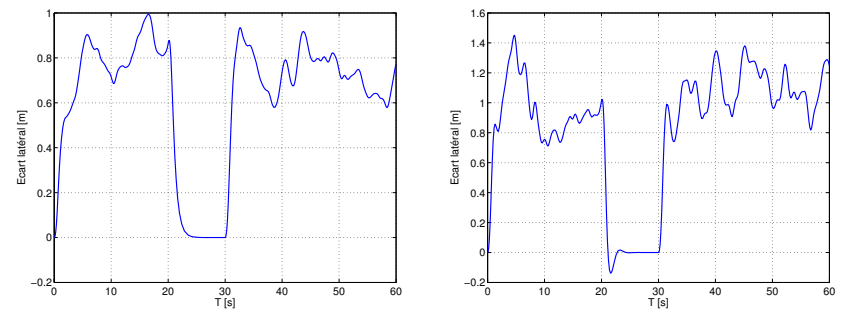

Fig. 6. Lateral displacement for a vehicle driving at $15 \mathrm{~m} . \mathrm{s}^{-1}$ (left) and $30 \mathrm{~m} . \mathrm{s}^{-1}$ (right), wind is masked between $20 \mathrm{~s}$ and $30 \mathrm{~s}$

has been generated by METEODYN society [Met05] in the frame of IRCAD project and is relevant to a lateral wind of $15 \mathrm{~m} . \mathrm{s}^{1}$.

Figures 3, 4 and 5 show variation of lateral displacement, of the road relative yaw angle and of the steering angle for a vehicle speed of 15 and $30 m . s^{1}$. Although the lateral speed is approximately constant, at the highest speed, the lateral control has problem to achieve a lateral correction sufficient to bring the vehicle back to a small lateral displacement.

In the second simulation, wind has been masked between $20 \mathrm{~s}$ and $30 \mathrm{~s}$. Figures 6,7 and 8 show the impact of a sudden lateral wind. The difference on lateral displacement is now clear: it is about $80 \mathrm{~cm}$ for a vehicle speed of 

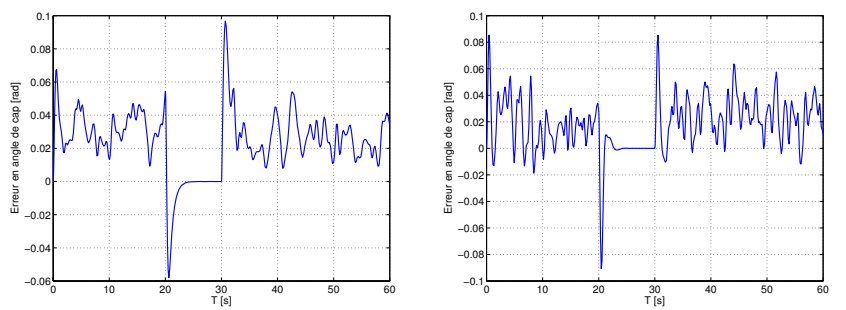

Fig. 7. Yaw angle error for a vehicle driving at $15 \mathrm{~m} . \mathrm{s}^{-1}$ (left) and $30 \mathrm{~m} . \mathrm{s}^{-1}$ (right), wind is masked between $20 \mathrm{~s}$ and $30 \mathrm{~s}$
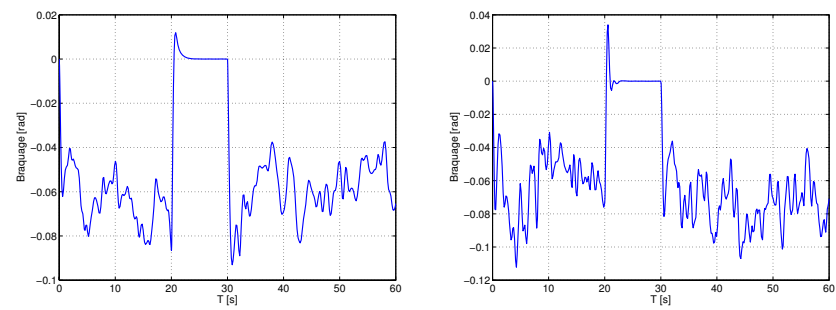

Fig. 8. Steering angle for a vehicle driving at $15 \mathrm{~m} . \mathrm{s}^{-1}$ (left) and $30 m . s^{-1}$ (right), wind is masked between $20 s$ and $30 s$

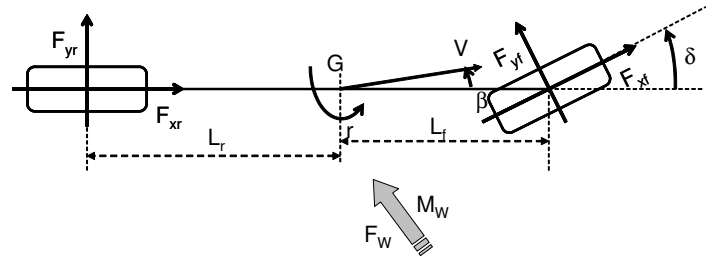

Fig. 9. Bicycle model

$15 m . s^{1}$ and greater than $1 m$ at $100 m . s^{1}$. With such lateral displacement, the vehicle goes off lane.

\section{2 vehicle model for lateral wind estimation}

Our goal is to estimate, using an instrumented vehicle, the forces and torques generated by the wind on the vehicle. In order to achieve this, we suppose that we have access to the following measures:

- $r$ : the yaw rate of the vehicle. It is now a common measure available on car equipped with an $\mathrm{ESP}^{1}$

- $y_{l}$ and $\psi_{l}$ : the lateral displacement and the yaw angle error. These measures are less common, but are now measured on high class vehicle, for instance on the AFIL $^{2}$ system developed by the car manufacturer PSA.

Moreover, steering angle and vehicle speed is also supposed to be known. In order to build the estimator, we use a bicycle vehicle model with a linear tire road interaction, presented in figure 9. In this model, $V$ is the vehicle speed, $\beta$ the lateral sliding angle and $r$ the yaw rate. $F_{x i}$ and $F_{y i}$ are the force generated the tire road interface.

This model is extended with the lateral displacement, $y$, and the yaw angle error $\Delta \psi$. The state vector is then $[\beta, r, y, \Delta \psi]^{t}$. The road is supposed to be a straight line,

1 Electronic Stability Program

2 AFIL is a lane departure warning assistance however, it could be also estimated in the process [Mam06]. The force and torque generated by the wind are introduced in the system as an input $W=\left[F_{w}, M_{w}\right]^{t}$. The model is defined by the following equations:

$$
\left\{\begin{array}{l}
\dot{x}=A x+B u+B_{W} W \\
y=C x
\end{array}\right.
$$

The different matrixes are developed in the first annex. State is then extended with $W$, the system is now:

$$
\left\{\begin{array}{l}
\dot{X}=\hat{A} X+\hat{B} u \\
Y=\hat{C} X
\end{array}\right.
$$

Where:

$$
\hat{A}=\left[\begin{array}{cc}
A & B_{W} \\
0 & 0
\end{array}\right] \hat{B}=\left[\begin{array}{l}
B \\
0
\end{array}\right] \hat{C}=\left[\begin{array}{ll}
C & 0
\end{array}\right]
$$

\section{WIND ESTIMATOR}

Using model developed previously for the estimation, we have the estimated state $\hat{X}$ and the system:

$$
\left\{\begin{array}{l}
\dot{\hat{X}}=\hat{A} \hat{X}+\hat{B} u+M(\hat{Y}-Y) \\
\hat{Y}=\hat{C} \hat{X}
\end{array}\right.
$$

By doing the difference between the two systems, we can explain the error $e=\hat{X}-X$ with respect to the parameters of the system:

$$
\dot{e}=(\hat{A}+M \hat{C}) e
$$

We can ensure that the error converge towards 0 , setting all eigen values of $\hat{A}+M \hat{C}$ with a negative real part, with respect to $M$.

\subsection{Simulation and estimator architecture}

Data provided to the estimator are simulated through the four wheeled model developed in section 3.1. Longitudinal control objective is to remain at a constant speed. Lateral vehicle control ensure the lane keeping task. As shown in figure 10, data generated by the vehicle model go to sensor model blocks. Noise is added, accordingly to the sensor:

- Vision sensor: gaussian noise is added on both lateral displacement and yaw angle error. The filter is set in order to have a $10 \mathrm{~cm}$ error at $3 \sigma$ on the lateral displacement and a $0.05 \mathrm{rad}$ error at $3 \sigma$ on the yaw angle error.

- Inertial sensor: a gaussian noise is also added in order to have a 0.01 rad.s $s^{-1}$ error at $3 \sigma$.

- Steering angle sensor: the measure generated by this sensor differs from previous ones. In fact, an optical coder delivers the absolute position of the coder. The main error comes from the translation from the numerical value to the angle. To model this sensor, the steering angle is divided by the resolution of the coder, rounded to the inferior integer and multiplied by the resolution. 


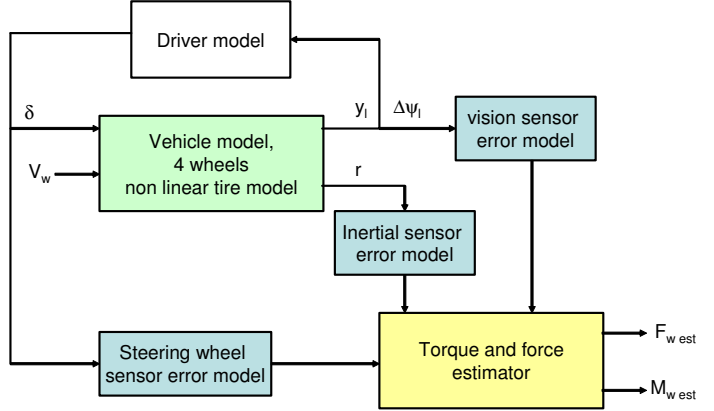

Fig. 10. Estimator architecture

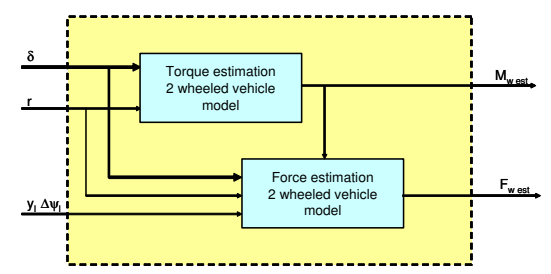

Fig. 11. Estimation of force and torque generated by the wind using two observers

\subsection{Observability and numerical problems}

Observability of the system 6 has been validated in theory, by computing the rank of the observability matrix $O^{3}$ which is 6 . So, the system is observable. Nevertheless, the system is extremely bad shaped. In fact, the lower eigen values of the system is about $10^{-6}$ and the highest around $10^{5}$. Even under normalized conditions, the dynamic of the system is too slow to perform both estimation. Simultaneous observation of the force and torque is not possible. However, as we have a direct measure of the yaw rate, we can estimate the torque generated by the wind quite well. This leads us to a separate estimation of torque and force generated by the wind on the vehicle.

\subsection{Force and torque estimation using two estimators}

As the observability has been proved in theory and given that the estimation of the torque is numerically possible, two observers have been linked to estimate both components of the wind.

First one will estimate the torque generated by the wind, it will use as input the yaw rate, the steering angle and the vehicle speed. It is observable, with respect to the previous development. Estimated torque is then given as an input for the second observer that will use previous inputs and the lateral displacement. The figure 11 summarizes this double estimator.

The results of the estimators are good, as shown on figure 12 and 13 . We estimate wind force and torque according to the two previous simulations. The error on the estimation remains small: it is smaller than $300 \mathrm{Nm}$ for values of torque between $2000 \mathrm{Nm}$ and $5000 \mathrm{Nm}$. The error on the force is larger, as the filter is 1s late. But we can not more constrain the estimator as it will become more and more subject to noises and become unstable.

${ }^{3} O=\left[\hat{C} \hat{C} \hat{A} \hat{C} \hat{A}^{2} \hat{C} \hat{A}^{3} \hat{C} \hat{A}^{4} \hat{C} \hat{A}^{5}\right]^{t}$
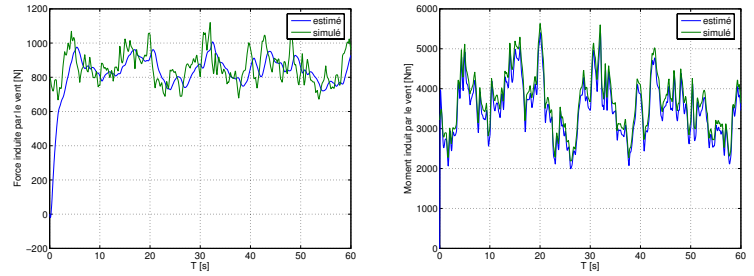

Fig. 12. Wind force and torque estimation on the first simulation
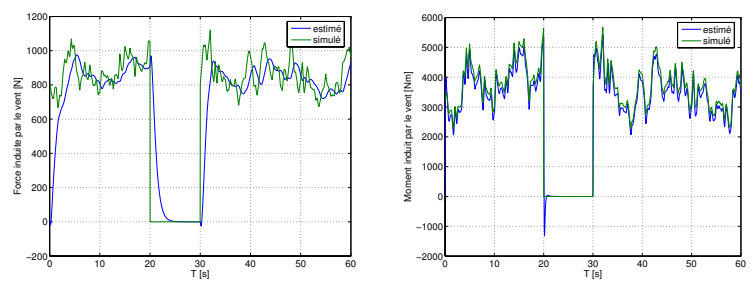

Fig. 13. Wind force and torque estimation on the second simulation

\subsection{Speed variation and estimator gain}

Model used to develop the estimator is a bicycle model. One of the hypothesis of this model is a constant speed, and the system 6 is used in order to determine the gain. However, it is possible to apply the developed method and to adapt it for a speed varying vehicle using a gain scheduling method [Sti99].

The gain matrix $M$ is computed for a given speed interval and is named accordingly to the center of the interval $M_{V i}$. When the speed of the vehicle corresponds to a single gain, this is the final gain of the system. When interval are overlapping, the resulting gain is computed accordingly to the position of the speed with respect to the intervals.

\section{DRIVER ASSISTANCE}

The goal of this article is to develop a driving assistance that warns the driver in case of excessive wind. In the previous section, we have developed a virtual sensor able to estimate the force and torque generated by the wind on the vehicle. The problem is now to give the information to the driver.

As the driver must act on the steering wheel in order to control perturbation induced by the wind, he mobilizes a part of tire road friction in order to remain on the lane. The available road friction is limited, we choose this quantity in order to define a danger regarding the wind.

In order to reject the perturbation generated by the wind, the driver must generate at the tire road interface the following force (according to system 3 ):

$$
\left\{\begin{array}{l}
F_{f}=-\frac{M_{w}+L_{r} F_{w}}{L_{f}+L_{r}} \\
F_{r}=\frac{M_{w}-L_{f} F_{w}}{L_{f}+L_{r}}
\end{array}\right.
$$

Moreover, the driver must generate an acceleration in order to keep a constant speed, we call the force generated at the tire road interface $F_{m}$. We now have the expression 


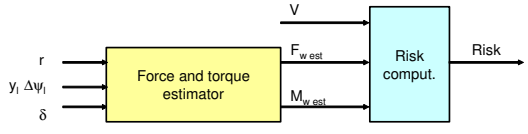

Fig. 14. Architecture of driver warning assistance

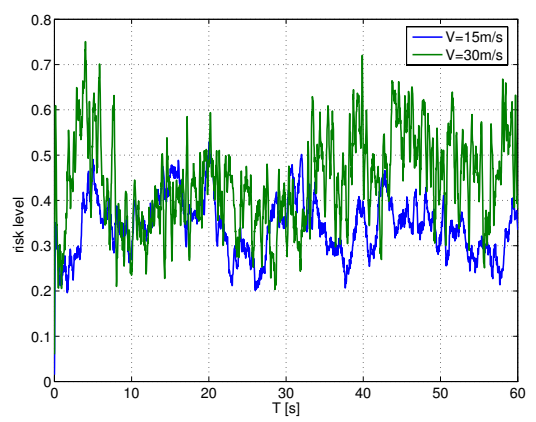

Fig. 15. Risk level for $V=15 \mathrm{~m} / \mathrm{s}$ and $V=30 \mathrm{~m} / \mathrm{s}$

of the mobilized friction to counter the wind effect on the front and rear tire:

$$
\mu_{m o b_{f}}=\frac{\left(\frac{M_{w}+L_{r} F_{w}}{L_{f}+L_{r}}\right)^{2}+F_{m}^{2}}{M g \frac{L_{r}}{L_{f}+L_{r}}}
$$

and

$$
\mu_{m o b_{r}}=\frac{\left(\frac{M_{w}-L_{f} F_{w}}{L_{f}+L_{r}}\right)^{2}}{M g \frac{L_{f}}{L_{f}+L_{r}}}
$$

The expression of $F_{m}$ is:

$$
F_{m}=-F_{\text {wlongi }}=-\frac{1}{2} C_{x}(\sigma) \rho S V_{r}^{2}
$$

For a passenger car, this force remains low: at $50 \mathrm{~km} / \mathrm{h}$, with a direct front wind of the same speed, the force is below $500 N$ and far below lateral contribution. In the following, we neglect this force.

Risk level is then computed according to the figure 14.

The risk is then computed for a vehicle that drives at $15 \mathrm{~m} / \mathrm{s}$ and $30 \mathrm{~m} / \mathrm{s}$. Risk levels are presented on the figure 15, using equations 9,10 and the developed estimator architecture. For $V=15 \mathrm{~m} / \mathrm{s}$ the average risk is 0.34 . At $V=30 \mathrm{~m} / \mathrm{s}$, the average risk is 0.49 . This risk level directly matches a mobilized friction, which in the second case is high. According to [Gla03], we have set a scaled warning for a mobilized friction corresponding to 0.3, 0.4 and 0.5 .

\section{CONCLUSION}

In this article we have developed a method to evaluate the wind threat on the vehicle dynamic.

First goal was to measure the impact of the wind on the lateral dynamic. An observer based method has been developed to achieved the measure of force and torque generated by the wind on the vehicle. As a one step estimation has numerically failed, but the observability was proved, a two steps observers has been implemented and gives good results.
Next, the risk associated to the wind has been matched with the friction needed to correct the trajectory. It is the indicator used in order to evaluate the threat and give warning to the driver.

Further development of this assistance will need to be both evaluated and transposed to a real vehicle in order to achieve the complete validation of the system with a driver in the loop process.

The evaluation of the algorithm could be carried out in an open environment as a straight road with localized blower on a side.

\section{REFERENCES}

[Eld06] Andrew Mark Eldredge Improved State Estimation for Miniature Vehicle thesis, Brigham Young University, 2006.

[Kum05] M. Kumon, I. Mizumoto, Z. Iwai and M. Nagata Wind Estimation by Unmanned Air Vehicle with Delta Wing IEEE ICRA 2005, Barcelona, Spain

[Cor04] R. Cortesao, N. Bajcinca Model Matching control for steer by wire vehicles with under actuated structure IEEE IROS 2004, Sendai, Japan

[Bak87] C.J. Baker, Measures to control vehicle movement at exposed sites during windy periods Journal of Wind Engineering and Industrial Aerodynamics, 25, (1987) p151-161

[Net03] M. Netto, R. Labayrade, S.S. Ieng, B. Lusetti, J.M. Blosseville and S. Mammar Different modes on shared lateral control 10th ITS Wordl Congress, Madrid, Spain, 2003

[Gla07] S. Glaser, L. Nouveliere, B. Lusetti Speed Limitation Based on an Advanced Curve Warning System IEEE Intelligent Vehicles Symposium Istambul, Turkey 2007

[Dug77] H. Dugoff, P.S. Fancher, L. Segel An analysis of Tire Traction properties and their influence on Vehicle dynamic performance SAE Paper 700377

[Met05] Margarita Dufresne and Didier Delaunay, Effet des vents latéraux sur les véhicules routiers - Calcul des efforts et moments aérodynamiques IRCAD Project, WBS 1.7, 25/08/05

[Mam06] S. Mammar, S. Glaser, M. Netto Time to line crossing for lane departure avoidance: a theorical study and an experimental setting IEEE Transactions on Intelligent Transportation System vol7 no2 2006

[Sti99] D.J. Stilwell and W.J. Rugh Interpolation of Observer state feedback controllers for gain scheduling IEEE Transactions on Automatic Control vol44 no6 1999

[Gla03] S. Glaser and V. Aguilera. Vehicle infrastructure driver speed profile : towards the next generation of curve warning system 10th ITS World Congress, Madrid, Spain, 2003

\section{Appendix A. MODEL MATRIXES}

This section develops the matrixes of the model presented by the equation 3 . 


$$
\begin{aligned}
& A=\left[\begin{array}{cccc}
-2 \frac{C_{f}+C_{r}}{M V} & -1+\frac{2 L_{r} C_{r}-2 L_{f} C_{f}}{M V^{2}} & 0 & 0 \\
\frac{2 L_{r} C_{r}-2 L_{f} C_{f}}{I_{z}} & -\frac{2 L_{r}^{2} C_{r}+2 L_{f}^{2} C_{f}}{I_{z} V} & 0 & 0 \\
V & 0 & 0 & V \\
0 & 1 & 0 & 0
\end{array}\right] \\
& B=\left[\begin{array}{c}
2 \frac{C_{f}}{M V} \\
2 \frac{C_{f}}{I_{z}} \\
0 \\
0
\end{array}\right] B_{W}=\left[\begin{array}{cc}
\frac{1}{M V} & 0 \\
0 & \frac{1}{I_{z}} \\
0 & 0 \\
0 & 0
\end{array}\right] \\
& C=\left[\begin{array}{llll}
0 & 1 & 0 & 0 \\
0 & 0 & 1 & L \\
0 & 0 & 0 & 1
\end{array}\right]
\end{aligned}
$$

In these matrixes, the parameters are given by table A.1. $V$ is the vehicle speed, in $m . s^{-1}$.

\begin{tabular}{||c|c|c||}
\hline \hline name & parameter & units \\
\hline \hline$L_{f}$ & distance from CG to front axle & $\mathrm{m}$ \\
$L_{r}$ & distance from CG to front axle & $\mathrm{m}$ \\
$M$ & vehicle mass & $\mathrm{kg}$ \\
$I_{z}$ & vehicle inertia on z axe & $\mathrm{kg} \cdot \mathrm{m}^{2}$ \\
$C_{f}$ & front tire lateral stiffness & $\mathrm{N}$ \\
$C_{r}$ & rear tire lateral stiffness & $\mathrm{N}$ \\
$L$ & distance of measurement of lateral displacement & $\mathrm{m}$ \\
\hline \hline
\end{tabular}

Table A.1. Parameters of vehicle model 